BUTP-01/24

\title{
Pair of null gravitating shells I. Space of solutions and its symmetries
}

\author{
P. Hájiček and I. Kouletsis \\ Institute for Theoretical Physics \\ University of Bern \\ Sidlerstrasse 5, CH-3012 Bern, Switzerland
}

December 2001

\begin{abstract}
The dynamical system constituted by two spherically symmetric thin shells and their own gravitational field is studied. The shells can be distinguished from each other, and they can intersect. At each intersection, they exchange energy on the Dray, 't Hooft and Redmount formula. There are bound states: if the shells intersect, one, or both, external shells can be bound in the field of internal shells. The space of all solutions to classical dynamical equations has six components; each has the trivial topology but a non trivial boundary. Points within each component are labeled by four parameters. Three of the parameters determine the geometry of the corresponding solution spacetime and shell trajectories and the fourth describes the position of the system with respect to an observer frame. An account of symmetries associated with spacetime diffeomorphisms is given. The group is generated by an infinitesimal time shift, an infinitesimal dilatation and a time reversal.
\end{abstract}




\section{Introduction}

A remarkable feature of gravitation is that of gravitational collapse. The theory predicts spectacular stages of collapse such as the formation of black holes and the inevitability of a final singularity if a black hole is formed. There is some evidence that black holes indeed exist. Alas, the singularity means that the classical theory breaks down.

In this situation, it is natural to look to quantum theory for a remedy. However, a quantum theory of gravity as a logically self-consistent theory backed up by observation and experiment does not exist, and it seems unlikely that it will exist as such a theory in the near future. This provides our motivation first for focusing on a particular, truly physical, problem such as gravitational collapse and, second, for working with simplified models. Such models can give useful hints even before a full-fledged quantum gravity is invented. One may even compare them to the theory of atomic spectra before the invention of quantum electrodynamics. By simplifying technicalities, they enable us to construct exact quantum theories instead of just, say, semi-classical approximations thereof. In fact, no semi-classical approximation would do near the singularities.

Of course, if we reject semi-classical approximations as the leading idea then we are in for trouble. First, some semi-classical approximation must indeed be valid somewhere. At the very least this should be near the infinity, where the fields and interactions are weak, but it may even be as far down as near the black hole horizons of large astronomical objects, as current observations seem to be bearing out. We must thus confirm that the constructed theory does not have a wrong classical limit. Second, a semi-classical approach can always be formulated as a theory on a fixed classical potential. For gravity, this effectively boils down to a quantum theory on a fixed classical background spacetime. That has a great advantage: the conceptual difficulties of quantum gravity can be avoided. If we abandon semiclassical approximation then we are exposed at once to these problems.

Our method to meet conceptual problems is based on a suitably chosen set of gauge-invariant quantities. The quantum theory is then formulated exclusively in terms of these quantities. Since the gauge group in question is the diffeomorphism group of the whole spacetime, gauge invariance includes the property of being an integral of motion; these quantities are called Dirac observables. The use of Dirac observables in quantum gravity goes back to Bergmann [1], who also articulated two difficulties associated with them: First, there seemed to be no no-trivial dynamics' and, second, no single Dirac observable was known for general relativity (in general). A third difficulty has been discovered recently [2]: No Dirac observables exist in

\footnotetext{
1 "Frozen dynamics" was Bergmann's term.
} 
general relativity that are local functionals of the fields.

However, if the physical problem at hand can be given the form of a scattering problem so that every relevant question can be answered by a measurement in the asymptotically flat region of spacetime, then the above difficulties can be solved. This has been outlined in [3] for the case of quantum field theory on curved spacetime. Indeed, the gauge group of asymptotically flat spacetime does not include all diffeomorphisms but only those that do not move points at infinity. The diffeomorphisms that move such points are not gauge, but symmetry transformations. There is then enough symmetry to construct a non-trivial dynamics of Dirac observables [4]. Moreover, there are even two complete sets of Dirac observables, which are the usual asymptotic in- and out-fields of scattering theory. For gravity, these observables have been called "asymptotic invariants" by DeWitt [5] within a perturbative scattering theory and "radiative modes" by Ashtekar [6] within his exact theory of "asymptotic quantization". Finally, asymptotic fields are local in their corresponding asymptotic regions, and there is no necessity to write them as (non-local) functionals of the fields at finite times?

In order to construct a quantum theory based on Dirac observables, Poisson brackets within the chosen set of observables must be known: the algebra of Dirac observables. We shall calculate this algebra by specifying a gauge. The method has been invented by Kuchař [7] and fully developed by Hájičce and Kijowski [8]. It is based on the so-called Kuchar decomposition: all variables are split into Dirac observables describing the physical degrees of freedom, the embedding variables that represent the gauge variables and the embedding momenta that represent the dependent variables. The notions of background manifold and of covariant gauge fixing (necessary for the definition of embedding variables and for making calculations) are explained in [8].

This program has been successfully completed for a simplified collapse model, see [9] and [10]. It consists of a single spherically symmetric shell of null dust surrounded by its own gravitational field. The constructed quantum dynamics has been unitary; the shell wave packet has been sent from infinity by some observers, it has collapsed, bounced, re-expanded and has been finally captured by the same observers at infinity. For sufficiently high energy of the packet, most of the packet has passed the Schwarzschild radius back and forth. This does not lead to contradiction because the quantum Schwarzschild radius has been black (black hole) when the shell has been passing it inwards, and it has been white (white hole) when the shell has been passing it outwards.

There have been, however, some questions. First, the shell has a zero radial

\footnotetext{
${ }^{2}$ Of course, the $S$-matrix is non local, as one could see if one managed to write it in $x$ representation, but this does not seem to cause problems.
} 
extension and the spacetime under it is Minkowski spacetime. The canonical formalism can be cast in the form of a shell moving on Minkowski spacetime [11]. It is then very simple and in fact natural to arrange the bounce! But what happens if the system has non-zero radial extension? Then the dynamics cannot very naturally be reformulated as motion on Minkowski spacetime. Can a unitary quantum mechanics still be constructed?

Second, what is the nature of the metric outside the shell? For a quantum shell in a spherically symmetric case, the metric belongs to dependent variables so it must be a quantum metric. However, the notion of such a quantum metric is not gauge invariant [10]. Even classically, what could be the role, say, of an "average" metric at a point of the background manifold, even if a gauge choice makes such notion well-defined? The problem is that in order for a tensor field to be a metric it must have signature +2 . Since the signature is not a linear property, the sum of two metrics need not be any metric at all. The analogous notions of an "operator of metric" at a given point of the background manifold and of its expectation value in some quantum state do not make much sense either; they are not useful quantities for the description of the quantum field outside the shell. How can we then obtain any physically meaningful information about the field outside the shell?

The two questions provide our motivation for the study of two-shells: It may be the simplest system that has a non-zero extension; the second shell moves in the field of the first one, that is, classically, in a Schwarzschild spacetime. It seems then that there is no possibility to reduce everything to a motion on Minkowski spacetime. Hence, the first question can be studied. Moreover, the second shell probes the field of the first one. In a spherically symmetric case, this is even literally so because any shell influences only the part of spacetime that lies outside of it. If we manage to construct a quantum theory, we may read from the asymptotic state of the out-going second shell information about the field of the first one.

As yet, however, no quantum theory has been constructed. Only the classical part of the work has been done: some complete (phase space spanning) sets of Dirac observables have been found and their Poisson brackets have been calculated. The calculation requires several steps so we have split it into three papers, numbered I, II and III. The derivation of the results is described in such a way that it is easy to generalize it to any number of shells, and the results themselves are formulated so that their extension to any number of shell is trivial.

The present paper, I, describes and parametrizes the set of all solutions. It turns out that two shells form a very interesting and much less trivial system than a single shell. The reason is that two shells can intersect each other, and they interact in a relatively complicated way at the intersection. Physically, the interaction can be defined as Raychaudhuri's effect: positive matter crossing a null hypersurface 
enlarges the convergence of its null generators in dependence of the matter density. Thus, an out-going shell is left less divergent and so its energy is smaller after the crossing, while the in-going shell is made more convergent and so has a larger energy after the crossing. Technically, everything is determined for the shells by the requirement that the metric is continuous even at the crossing point. The resulting condition in the spherically symmetric case has been written down by Dray and 't Hooft [13] and by Redmount [14]. The interaction has a contact character. It is interesting that, if the shells intersect, the out-going shell can be left in a bound state after the crossing, while the in-going shell can be bound before the crossing ("bound" means that it does not reach infinity). All this gives a lot of structure to the space of solutions. An intriguing question (that we don't answer in these three papers) is whether a true Hamiltonian can be written down that contains the interaction at the crossing.

Finally, the present paper also specifies the symmetries of the two-shell system. They are the diffeomorphisms that move points at infinity and are compatible with the spherical symmetry of the model. They can help us to construct the time evolution as shown in [4].

The plan of the paper is as follows. Sec. 2 describes the construction of all possible solutions to Einstein equations that are spherically symmetric, contain two null shells and no other sources of gravity. The space of all such solutions consists of six disconnected subspaces. Each subspace has the trivial topology but a non-trivial boundary. Coordinates we choose to parametrize the solutions in different subspaces can be separated into two groups: geometric and frame-position parameters. The geometric parameters determine the metric and the shell trajectories up to isometry. The frame-position parameters define the position of the system with respect to an (asymptotic) observer frame.

Sec. 3 presents an example of gauge choice for one of the subspaces. The set of spacetimes is turned to a set of metric fields and shell trajectories on a background manifold. In Sec. 1 , the symmetries of the space of solutions are specified. They consist of time shifts, time reversal and dilatations (re-scalings). The gauge defined in Sec. 3 is employed for the calculation of the action of the symmetries on embedding variables; this action is important for the construction of the dynamics as described in [4].

\section{Space of solutions}

Spherically symmetric solutions with two null shells are well-known, cf. [12, 13 and [14]. In this section, a standardized construction of these solutions is described. The construction depends on certain parameters. If some of these parameters change, 
the physical properties of the solution also change. Such parameters can be chosen as coordinates in the physical phase space. Some other parameters change but the physical properties remain the same. Such parameters describe pure gauge. A bijective association of all solutions with the values of four parameters lying in certain spaces will be established.

All spacetime solutions can be constructed using the following rules. The metric in any shell-free part of the spacetime is the Schwarzschild one corresponding to some value $M$ of the Schwarzschild mass parameter. We assume that the innermost part is flat $(M=0)$ and contains a regular center so that Cauchy hypersurfaces have the topology of $\mathbf{R}^{3}$. The shells form hypersurface boundaries of these pieces of Schwarzschild spacetimes. The hypersurfaces are light-like with respect to the metrics of their neighboring spacetimes. There must be coordinates in a neighborhood of any shell point such that the metric in the neighborhood is continuous [12. This holds even for the crossing points of two shells, see [13] and [14. Using these rules, we obtain all solutions. The energy density of the shell matter is assumed to be positive.

The two shells are physically distinguishable. Different shells are described by a parameter $s=1,2$. These indices represent the properties of the shell material by which we can recognize them. One can also imagine that the first shell is green and the second red. However, the shell dynamics is invariant with respect to shell permutation.

The assumption on the topology of Cauchy hypersurfaces implies that there is only one infinity, that is only one scri, $\mathcal{I}$. We assume that the observers live there and define a particular reference frame. With respect to these observers, each shell is either out- or in-going (irrespectively of whether the shell reaches the $\mathcal{I}$ or not). This property of a shell is described by the parameter $\eta: \eta=+1$ if the shell is out-going and $\eta=-1$ otherwise. Since the shells are distinguishable we have to consider six cases:

A: Both shells are in-going, $\eta_{1}=\eta_{2}=-1$, with the first shell on the left.

A': Both shells are in-going, $\eta_{1}=\eta_{2}=-1$, with the second shell on the left.

B: Both shells are out-going, $\eta_{1}=\eta_{2}=+1$, with the first shell on the left.

B': Both shells are out-going, $\eta_{1}=\eta_{2}=+1$, with the second shell on the left.

C: The first shell is in- and the second is out-going, $\eta_{1}=-\eta_{2}=-1$.

C': The first shell is out- and the second is in-going, $\eta_{1}=-\eta_{2}=+1$.

Our construction will now be outlined case by case. 


\subsection{Parallel shells}

Cases A and A' are schematically depicted by Fig. 1; case A' differs from A just by swapping the shells; we can deal with both cases simultaneously by speaking about internal and external shells. The intershell spacetimes are denoted by $\mathcal{M}_{K}$, $K=l, m, r$, where the subscripts stand for left, middle and right. The Schwarzschild mass of $\mathcal{M}_{K}$ is denoted by $M_{K}$. The mass of $\mathcal{M}_{l}$ is zero. $M_{r}$ is the total mass of the system and $M_{m}$ is the total energy of the first shell. The energy of the second shell is $M_{r}-M_{m}$.

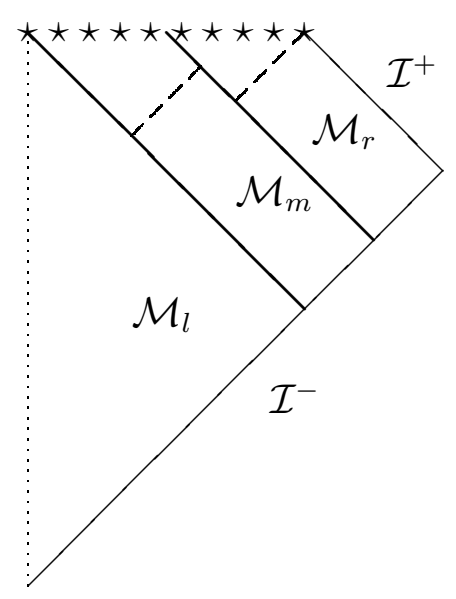

Figure 1: Penrose diagram of case A. The star line is the singularity, the dotted line is the regular center, the thick lines are the shells and the dashed lines are the Schwarzschild horizons. Case B can be considered as the time reversal of case A.

Any solution of this type with metric and shell trajectories can be constructed as follows. Let $\overline{\mathcal{M}}_{K}$ be the maximal analytic extension of the spacetime $\mathcal{M}_{K}$. The quotient $\overline{\mathcal{M}}_{K} / \mathrm{SO}(3)$ of these spherically symmetric spacetimes are themselves spacetimes, albeit two-dimensional. The spacetimes $\overline{\mathcal{M}}_{K} / \mathrm{SO}(3)$ can be given time and space orientations. We assume that the orientation of pasted spacetime agrees with the orientation of its parts. The notions of the out- and in-going shell can also be defined more precisely: future oriented tangential vector is oriented in any orthonormal two-bein to the right (left) for out- (in-)going shell. In this way, the orientation of the shell motion is indeed well defined even if the shell does not intersect infinity.

The orientation of Minkowski space $\left(M_{l}=0\right)$ deserves a special attention. There are two physically different space orientations of it: that with the infinity right and the center left and the opposite one. A shell spacetime constructed from a piece of Minkowski spacetime oriented in the latter way and a piece of Schwarzschild 
spacetime is shown in Fig. 2. However, the topology of Cauchy surfaces of the spacetime shown in Fig. 2 is $\mathbf{R} \times S^{2}$ instead of $\mathbf{R}^{3}$ as required, and the energy density of the shell there must be negative.

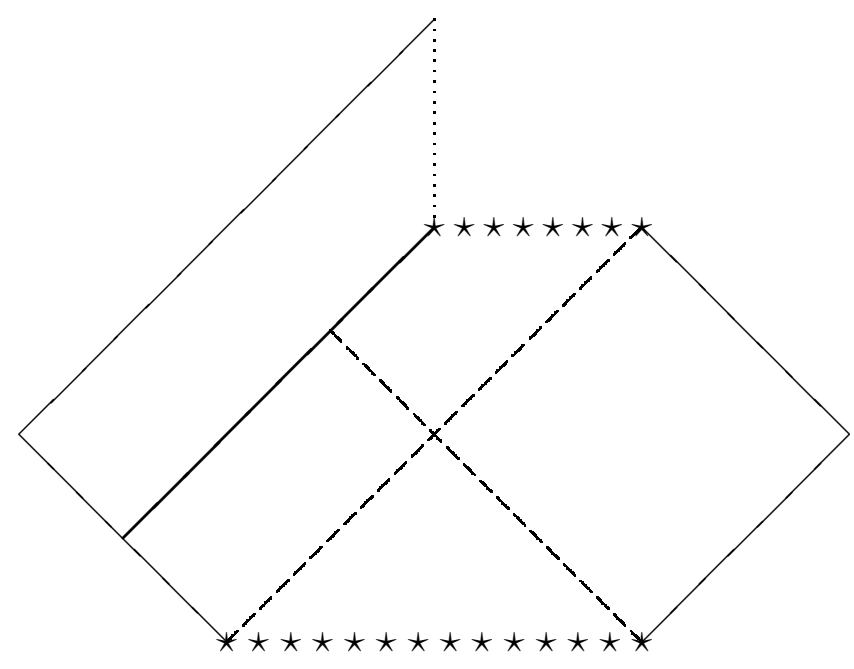

Figure 2: Penrose diagram of a shell spacetime containing a piece of Minkowski spacetime with the second orientation. The star lines are the singularities, the dotted line is the regular center, the thick lines are the shells and the dashed lines are the Schwarzschild horizons.

This follows from a Lemma shown in [13]:

Lemma 1 Suppose that i) a null shell forms a common boundary of two Schwarzschild spacetimes, ii) the spacetime with mass $M_{2}$ lies to the right and that with $M_{1}$ to the left, and iii) the shell has positive energy density. Then either the shell intersects $\mathcal{I}^{+} \cup \mathcal{I}^{-}$of the right scri in both spacetimes and

$$
M_{2}-M_{1}>0
$$

or the shell intersects $\mathcal{I}^{+} \cup \mathcal{I}^{-}$of the left scri in both spacetimes and

$$
M_{2}-M_{1}<0
$$

or the shell does not intersects $\mathcal{I}^{+} \cup \mathcal{I}^{-}$anywhere and

$$
M_{2}-M_{1}=0
$$

We assume, therefore, that the Minkowski spacetime is always oriented so that the regular center of the constructed spacetime is left, and the infinity is right. 
In $\overline{\mathcal{M}}_{K}, K=m, r$, the so-called Double-Null Eddington-Finkelstein (DNEF) coordinates are based on four functions $U_{K}^{ \pm}$and $V_{K}^{ \pm}$. The function $U_{K}^{\alpha}$ is defined by

$$
U_{K}^{\alpha}:=\alpha\left(T_{K}-R_{K}-2 M_{K} \ln \left|\frac{R_{K}}{2 M_{K}}-1\right|\right)
$$

for $\alpha= \pm 1$. The domain of $U_{K}^{+}$is the past of the out-going Schwarzschild horizon $H_{K}^{\text {out }}$, that of $U_{K}^{-}$the future of $H_{K}^{\text {out }}$. Similarly,

$$
V_{K}^{\beta}:=\beta\left(T_{K}+R_{K}+2 M_{K} \ln \left|\frac{R_{K}}{2 M_{K}}-1\right|\right),
$$

the domain of $V_{K}^{+}$being the future of the in-going Schwarzschild horizon $H_{K}^{\text {in }}$, and that of $V_{K}^{-}$the past of $H_{K}^{\text {in }}$. Any pair of such functions define coordinates in that quadrant of $\overline{\mathcal{M}}_{K}$ where their domains overlap. We denote these quadrants by the pairs of signs $(\alpha \beta):(++),(+-),(-+)$ and $(--)$.

The metric in $\mathcal{M}_{K}$ has the form

$$
d s^{2}=-A_{K} d U_{K} d V_{K}+R_{K}^{2} d \Omega^{2},
$$

where

$$
A_{K}=\left|1-\frac{2 M_{K}}{R_{K}}\right|=\alpha \beta\left(1-\frac{2 M_{K}}{R_{K}}\right)
$$

and, in each quadrant $(\alpha \beta)$,

$$
R_{K}=2 M_{K} \kappa\left[\alpha \beta \exp \left(\frac{-\alpha U_{K}^{\alpha}+\beta V_{K}^{\beta}}{4 M_{K}}\right)\right] .
$$

Here, $\kappa$ is the well-known Kruskal function defined by its inverse:

$$
\kappa^{-1}(x):=(x-1) e^{x} .
$$

Our motivation to work with these double-null coordinates is that, in terms of them, many expressions and calculations, especially in the canonical part of the formalism in papers II and III, significantly simplify. This may be due to the simple relation of the DNEF coordinates to the time at infinity.

Observe that the formulae (3)-(5) are all valid even if $M_{K}=0$ because

$$
\lim _{M \rightarrow 0} 2 M \kappa\left[\exp \left(\frac{-U+V}{4 M}\right)\right]=\frac{-U+V}{2} .
$$

There is then, of course, only one "quadrant", $(++)$.

The notation settled, let us turn to the construction proper. The first step is to choose the trajectory of the shell $S_{1}$ in $\overline{\mathcal{M}}_{l}$ which must be an in-going spherically symmetric null hypersurface. It is defined by the equation

$$
V_{l}^{+}=v_{l 1},
$$


where $v_{l 1} \in(-\infty, \infty)$ is a constant (the first parameter). The second step is to choose the trajectories of $S_{1}$ and $S_{2}$ in $\overline{\mathcal{M}}_{m}$. They must both be in-going null hypersurfaces, and so have the equations

$$
V_{m}^{+}=v_{m 1}
$$

and

$$
V_{m}^{+}=v_{m 2}
$$

where $-\infty<v_{m 1}<v_{m 2}<\infty$ are two further parameters. The third step is to choose the trajectory of $S_{2}$ in $\overline{\mathcal{M}}_{r}$ :

$$
V_{r}^{+}=v_{r 2}
$$

where $v_{r 2} \in(-\infty, \infty)$ is another parameter.

The fourth step is to cut the pieces $\mathcal{M}_{l}, \mathcal{M}_{m}$ and $\mathcal{M}_{r}$ along the chosen boundaries from $\overline{\mathcal{M}}_{l}, \overline{\mathcal{M}}_{m}$ and $\overline{\mathcal{M}}_{r}$ and paste them together to make the solution spacetime $\mathcal{M}$. The pasting is defined by the requirement that points with the same value of the Schwarzschild coordinate $R$ coincide. This is always (i.e., for any allowed values of $M_{m}, M_{r}, v_{l 1}, v_{m 1}, v_{m 2}$ and $\left.v_{r 2}\right)$ possible and unique, and it closes the construction.

The parameters chosen on the way are $\eta_{1}, \eta_{2}, M_{m}, M_{r}, v_{l 1}, v_{m 1}, v_{m 2}$ and $v_{r 2}$. Which of them describe physical properties of the solution and which of them are gauge? It is clear that $\eta_{1}, \eta_{2}, M_{m}$ and $M_{r}$ are physical, even geometric parameters. The parameter $v_{m 2}-v_{m 1}$ determines, together with $M_{m}$, the geometry between the two shells, so it is a geometric parameter, too.

The three parameters $M_{m}, M_{r}$ and $v_{m 2}-v_{m 1}$ determine the geometry of the solution uniquely. Indeed, all pieces $\mathcal{M}_{l}\left(v_{l 1}\right)$ cut from the Minkowski space $\overline{\mathcal{M}}_{l}$ along the curve $V_{l}^{+}=v_{l 1}$ for some value $v_{l 1} \in(-\infty, \infty)$ are isometric to each other; all pieces $\mathcal{M}_{m}\left(v_{m 1}, v_{m 2}\right)$ cut from $\overline{\mathcal{M}}_{m}$ with a fixed mass $M_{m}$ and difference $v_{m 2}-v_{m 1}$ are isometric, and all pieces $\mathcal{M}_{r}\left(v_{r 2}\right)$ are so too.

However, the position of the system with respect to a frame at infinity is also a physical parameter. In fact, $v_{r 2}$ is the value of the advanced time when the second shell is sent in; it is measurable by the observers near $i^{0}$ of $\mathcal{M}$ and can serve as the fourth observable. In fact, the variable $v_{r 2}-v_{m 2}+v_{m 1}$ is also a frame-position observable and can be chosen as one of our parameters instead of $v_{r 2}$.

In this way, we have arrived at four coordinates describing the physical phase space for case $\mathrm{A}\left(\eta_{1}=\eta_{2}=-1\right)$ :

$$
M_{m}, v_{m 2}-v_{m 1}, M_{r}, v_{r 2} .
$$

Their domains are:

$$
\begin{aligned}
M_{m} \in(0, \infty), & M_{r} \in\left(M_{m}, \infty\right), \\
v_{m 2}-v_{m 1} \in(0, \infty), & v_{r 2} \in(-\infty, \infty) .
\end{aligned}
$$


The remaining parameters $v_{l 1}$ and $v_{m 1}$ are then a kind of gauge.

The inequalities $M_{m}>0$ and $M_{r}>M_{m}$ are a consequence of the requirement that the energy density of the shells is positive (cf. [13]). The manifold structure of this part of the physical phase space, as well as the physical meaning of its points, are now both well-defined.

Case B can be considered as time reversal of case A. The spacetime $\mathcal{M}_{l}$ is flat and has a regular center $c$. The first shell hypersurface is $S_{1}$. The total energy of the shell is $M_{m}$, and it is simultaneously the mass parameter of the Schwarzschild spacetime $\mathcal{M}_{m}$. The second shell lies at $S_{2}$ and has total energy $M_{r}-M_{m}$. The Schwarzschild spacetime $\mathcal{M}_{r}$ has mass parameter $M_{r}$.

The construction of the solution is entirely analogous to that of case A. The shell trajectories are

$$
\begin{array}{ll}
U_{l}^{+}=u_{l 1}, & U_{m}^{+}=u_{m 1}, \\
U_{m}^{+}=u_{m 2}, & U_{r}^{+}=u_{r 2} .
\end{array}
$$

The physical parameters are

$$
M_{m}, u_{m 2}-u_{m 1}, M_{r}, u_{r 2},
$$

and they have domains

$$
\begin{aligned}
M_{m} \in(0, \infty), & M_{r} \in\left(M_{m}, \infty\right), \\
u_{m 2}-u_{m 1} \in(0, \infty), & u_{r 2} \in(-\infty, \infty) .
\end{aligned}
$$

The parameters $u_{l 1}$ and $u_{m 1}$ are again a kind of gauge.

\subsection{Crossing shells}

Cases C and C' can be tackled simultaneously if we work with out-going and in-going shells instead of $s$-numerated ones.

In this case, the shells have to intersect each other. This is a consequence of the assumption that a piece of Minkowski spacetime contains a regular center to the left. Hence, the shells separate each solution spacetime $\mathcal{M}$ in four subspacetimes, cf. Fig. 6 : The flat subspacetime $\mathcal{M}_{l}$ (left), the subspacetime $\mathcal{M}_{d}$ (down) with Schwarzschild mass $M_{d}$, the subspacetime $\mathcal{M}_{u}$ (up) with mass $M_{u}$, and the subspacetime $\mathcal{M}_{r}$ (right) with mass $M_{r}$. The shells start with total energies $M_{r}-M_{d}$ and $M_{d}$. After they cross at the point $O$ with the value $r$ of Schwarzschild radius, they change their total energies to $M_{u}$ and $M_{r}-M_{u}$. These parameters satisfy the relation

$$
r\left(r-2 M_{r}\right)=\left(r-2 M_{u}\right)\left(r-2 M_{d}\right) .
$$


Eq. (7) follows from the requirement of continuity at the crossing point $O$ and has been derived in [13] and [14]. The encounter leads to exchange of energy between the shells, some energy passing from the out-going to the in-going shell.

The construction of the solution in this case uses the same method as in the case A but is more complicated. There are now four intershell spacetimes $\mathcal{M}_{K}$, $K=l, u, d, r$, four (properly oriented) extensions $\overline{\mathcal{M}}_{K}$, three non-trivial masses, and the spacetimes $\mathcal{M}_{K}$ are wedges with vertices $O_{K}$ of radius $r$ rather than stripes. The arrangement of the spacetimes $\mathcal{M}_{K}$ around the vertex is illustrated by Fig. $⿴$.

In fact, the geometry of the solution is completely determined by the three masses $M_{u}, M_{d}$ and $M_{r}$ because Eq. (7) implies that

$$
r=\frac{2 M_{u} M_{d}}{M_{u}+M_{d}-M_{r}} .
$$

Important restrictions on the domains of the masses are

$$
M_{u}>0, \quad M_{d}>0, \quad M_{r}>0 .
$$

The first two are equivalent to the requirement that the energy density of the shells is positive. The last one guarantees that the total energy of the system is positive. This follows from the Positive Mass Theorem [15], the topology of Cauchy surfaces being $\mathbf{R}^{3}$, and the positivity of the energy density of the shells. Within this scenario, Eq. (8) implies

$$
M_{r}<M_{u}+M_{d} .
$$

Cases C, C' are different from cases A, A' and B, B' in that they contain bound states of the external shell in the field of the internal one. This manifests itself in the total mass of the external shell, $M_{r}-M_{u}$ or $M_{r}-M_{d}$ being negative. There are four generic subcases depending on whether both external shells are unbound, the out-going one is bound, the in-going is bound, or both are bound. Between bound and unbound cases, there are five boundary ones with one or both shells being marginally bound (lying at Schwarzschild horizons).

We shall denote the subcases of case $\mathrm{C}$ by $\mathrm{C}_{a b}$, where

$$
a:=\operatorname{sgn}\left(M_{r}-M_{u}\right), \quad b:=\operatorname{sgn}\left(M_{r}-M_{d}\right),
$$

so that the values of $a$ and $b$ are,+- or 0 . Case $C^{\prime}$ is geometrically identical to case $\mathrm{C}$, but the shells are swapped: the in-going shell becomes the second one. The position of different subcases in the physical phase space is illustrated by Fig. 3 .

Given the masses $M_{l}=0, M_{u}, M_{d}$ and $M_{r}$, the four Schwarzschild spacetimes $\overline{\mathcal{M}}_{l}$, $\overline{\mathcal{M}}_{u}, \overline{\mathcal{M}}_{d}$ and $\overline{\mathcal{M}}_{r}$ are determined. To perform the construction, we need to know how the wedges $\mathcal{M}_{l}, \mathcal{M}_{u}, \mathcal{M}_{d}$ and $\mathcal{M}_{r}$ are to be cut from them. The orientation of 


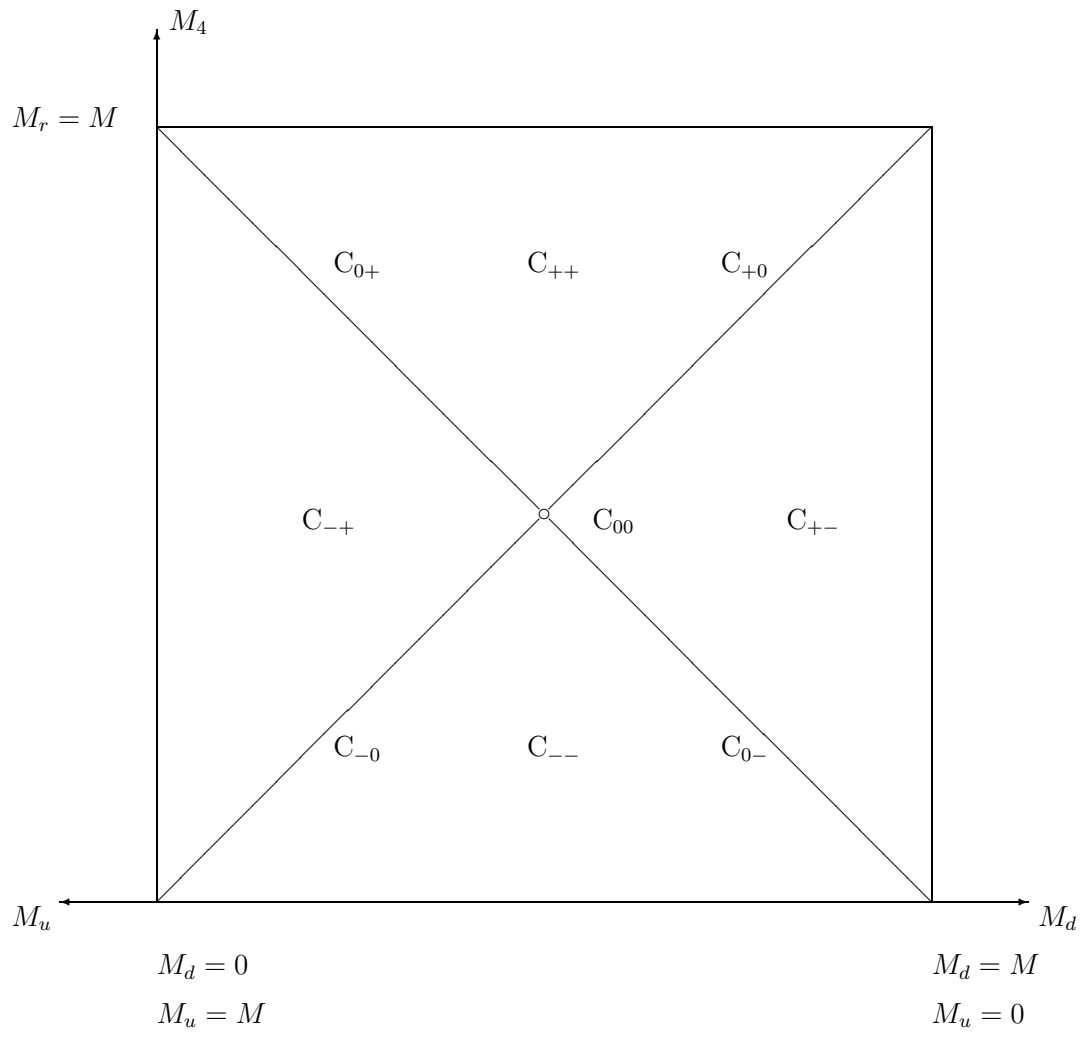

Figure 3: Cut $M_{u}+M_{d}=M$ through the positive octant of the $M_{u}, M_{d}$ and $M_{r}$ space

the wedges is fixed by their relative position with respect to the shells. Hence $\mathcal{M}_{l}$ opens left, $\mathcal{M}_{d}$ to the past, $\mathcal{M}_{u}$ to the future and $\mathcal{M}_{r}$ right. Then each wedge is determined just by the coordinates of its vertex.

To find in which quadrant of $\overline{\mathcal{M}}_{K}$ the vertex $O_{K}$ lies requires two steps. The first is based on Eq. (8). Two relations between the vertex radius $r$ and differences of masses follow from it:

$$
r-2 M_{r}=\frac{2\left(M_{r}-M_{u}\right)\left(M_{r}-M_{d}\right)}{M_{u}+M_{d}-M_{r}},
$$

and

$$
r-2 M_{K}=\frac{2 M_{K}\left(M_{r}-M_{K}\right)}{M_{u}+M_{d}-M_{r}}
$$

for $K=u, d$. Eq. (13) implies that $r>2 M_{d}$ if the in-going shell is unbound, and that $r<2 M_{d}$ if it is bound. Similarly, $r>2 M_{u}\left(r<2 M_{u}\right)$ implies unboundedness 
(boundedness) for the out-going shell. Eq. (12) requires that $r>2 M_{r}$ if none, or both, of the shells are bound, and that $r<2 M_{r}$ if just one shell is bound. Thus, only two quadrants from four remain to be eligible. To find the quadrant uniquely, we use the so-called "argument of matching divergences" in the second step.

At each spherically symmetric null hypersurface in Schwarzschild spacetime, the Schwarzschild radius either increases (divergence) or decreases (convergence) in future direction or it is a part of one of the horizons. More specifically, $U^{+}=$const and $V^{-}=$const always diverge and $U^{-}=$const and $V^{+}=$const always converge for finite values of the constants. If two null hypersurfaces are to be pasted together as in our construction, then they must either both converge or both diverge or both have zero divergence (lie on horizons).

The out-going shell in the past of the vertex $O_{l}$ must diverge in Minkowski space $\overline{\mathcal{M}}_{l}$ and hence also in $\overline{\mathcal{M}}_{d}$, and it therefore lies in the domain of the function $U_{d}^{+}$. But this domain contains only two quadrants, $(++)$ where $r>2 M_{d}$ and $(+-)$ where $r<2 M_{d}$. Consequently, $O_{d} \in(++)\left(O_{d} \in(+-)\right)$ if the in-going shell is unbound (bound). Similarly, $O_{u} \in(++)\left(O_{u} \in(-+)\right)$ if the out-going shell is unbound (bound). In the marginally bound cases, the vertices lie at the corresponding horizons.

Applying the argument of matching divergences to the wedge $\mathcal{M}_{r}$, we find that the in-going shell converges (diverges) if it is unbound (bound), and the out-going shell diverges (converges) if it is unbound (bound). This leads to $O_{r}$ lying in the quadrant $(++)$ if both shells are unbound, in $(-+)$ if the out-going shell is bound and the in-going one unbound, in $(+-)$ if the out-going shell is bound and the ingoing one unbound, and in (--) if both shells are bound. The resulting spacetimes are shown by Figs. 14 . The marginally bound cases are also included.

The construction is determined by specifying the coordinates of the four vertices $O_{K}$ in the spacetimes $\overline{\mathcal{M}}_{K}$. The parameters $u_{K}$ and $v_{K}, K=l, d, u, r$, are defined as follows. In all cases, $O_{l}$ has finite coordinates and $u_{l}=U_{l}^{+}$and $v_{l}=V_{l}^{+}$. For the vertex $O_{d}$, we always have $u_{d}=U_{d}^{+}$. On the other hand, if the in-going shell is unbound $v_{d}=V_{d}^{+}$, if it is bound $v_{d}=V_{d}^{-}$, and if it is marginally bound $V_{d}^{+}=-V_{d}^{-}=\infty$. Similarly for other vertices; we can summarize the definition of the parameters as follows. For case $\mathrm{C}_{a b}$ with $a \neq 0$ and $b \neq 0$, it holds that

$$
\begin{array}{ll}
u_{d}=U_{d}^{+}, & v_{d}=V_{d}^{b}, \\
u_{u}=U_{u}^{a}, & v_{u}=V_{u}^{+}, \\
u_{r}=U_{r}^{a}, & v_{r}=V_{r}^{b} .
\end{array}
$$

For the marginally bound cases, if $a=0$ then $U_{u}^{ \pm}$and $U_{r}^{ \pm}$diverge, and if $b=0$ then $V_{d}^{ \pm}$and $V_{r}^{ \pm}$diverge. The parameters $u_{d}$ and $v_{u}$ are always defined by $u_{d}=U_{d}^{+}$and $v_{u}=V_{u}^{+}$. 


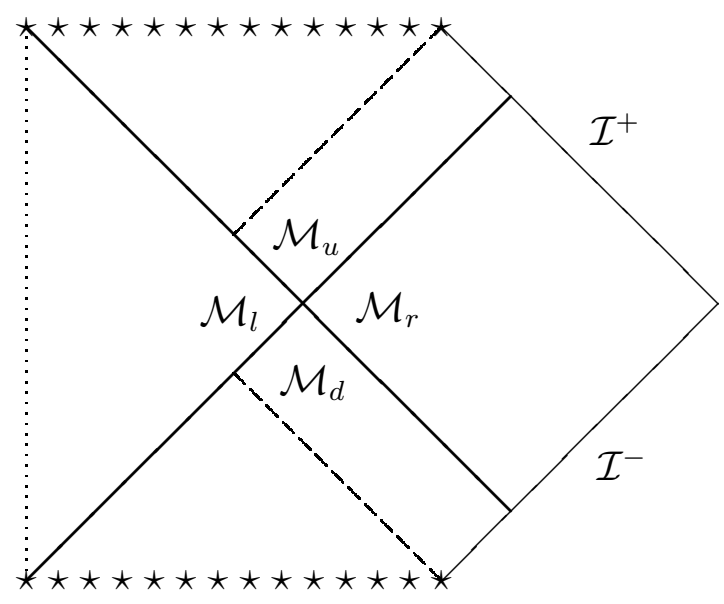

Figure 4: Penrose diagram of subcase $\mathrm{C}_{++}$. The dotted line is the regular center, the star lines are the singularities, the dashed lines are Schwarzschild horizons and the thick lines are the shells.

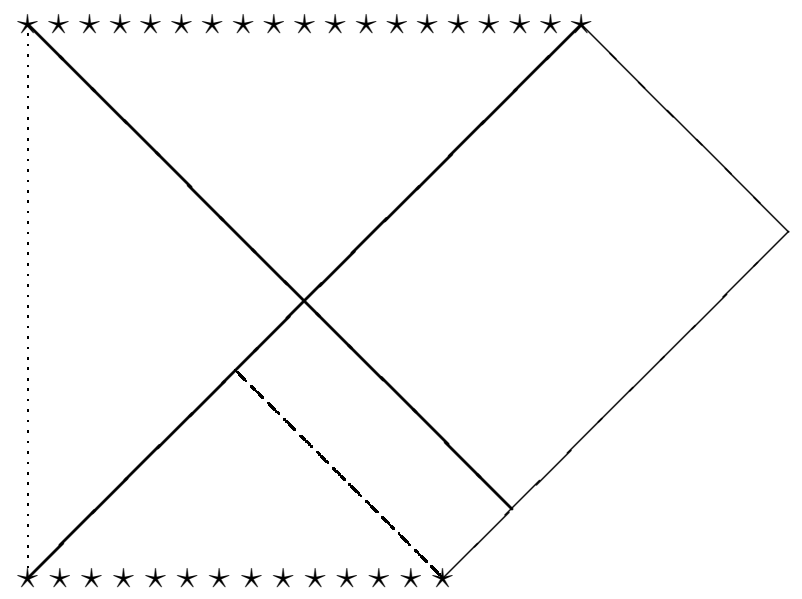

Figure 5: Penrose diagram of subcase $\mathrm{C}_{0+}$. The dotted line is the regular center, the star lines are the singularities, the dashed line is Schwarzschild horizon and the thick lines are the shells. Subcase $\mathrm{C}_{+0}$ can be considered as the time reversal of $\mathrm{C}_{0+}$. 


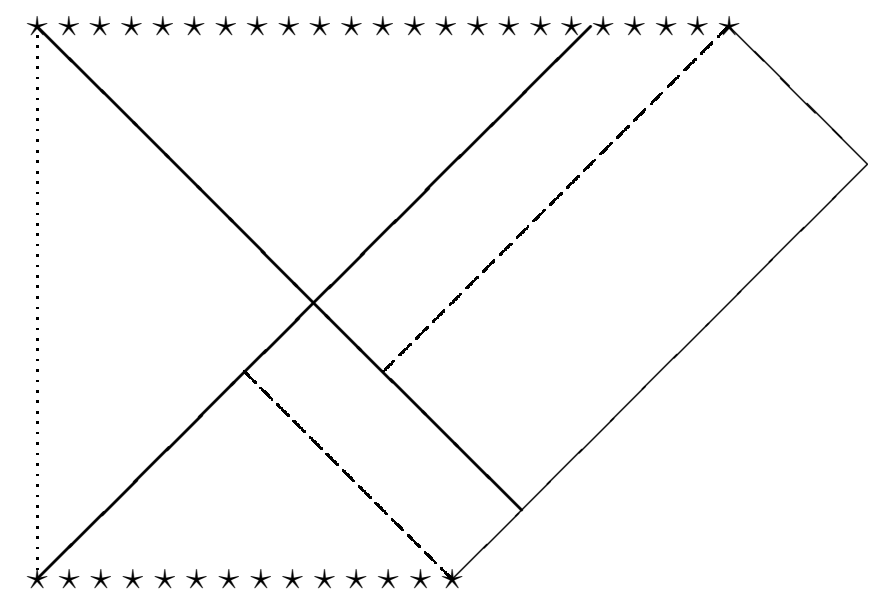

Figure 6: Penrose diagram of subcase $\mathrm{C}_{-+}$. The dotted line is the regular center, the star lines are the singularities, the dashed lines are Schwarzschild horizons and the thick lines are the shells. Subcase $\mathrm{C}_{+-}$can be considered as the time reversal of $\mathrm{C}_{-+}$.

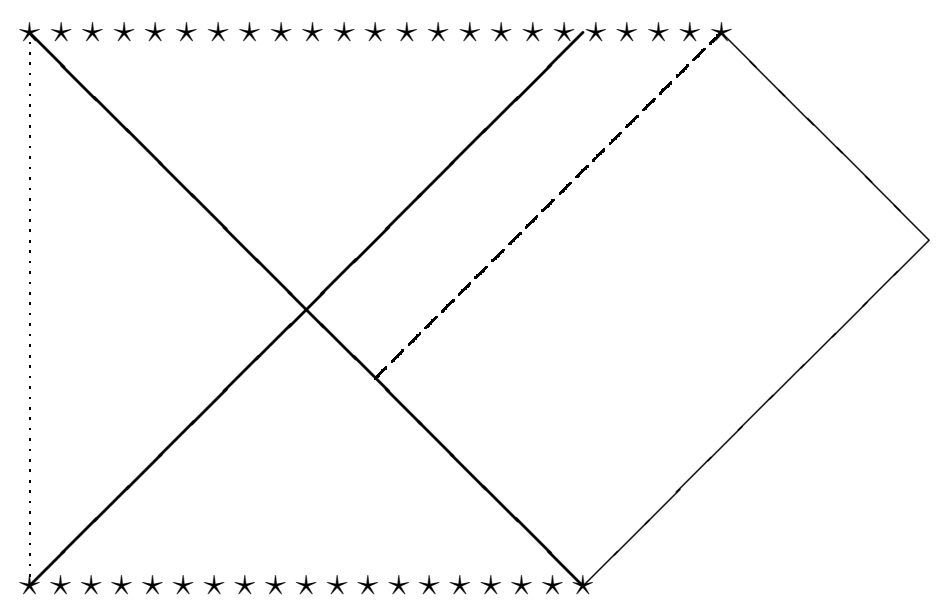

Figure 7: Penrose diagram of subcase $\mathrm{C}_{-0}$. The dotted line is the regular center, the star lines are the singularities, the dashed line is Schwarzschild horizon and the thick lines are the shells. Subcase $\mathrm{C}_{0_{-}}$can be considered as the time reversal of $\mathrm{C}_{-0}$. 


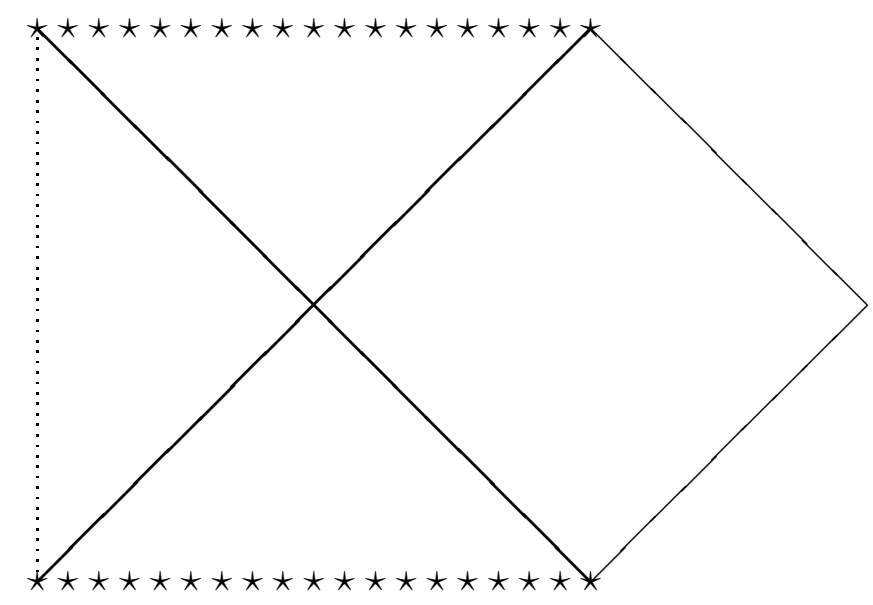

Figure 8: Penrose diagram of subcase $\mathrm{C}_{00}$. The dotted line is the regular center, the star lines are the singularities, and the thick lines are the shells.

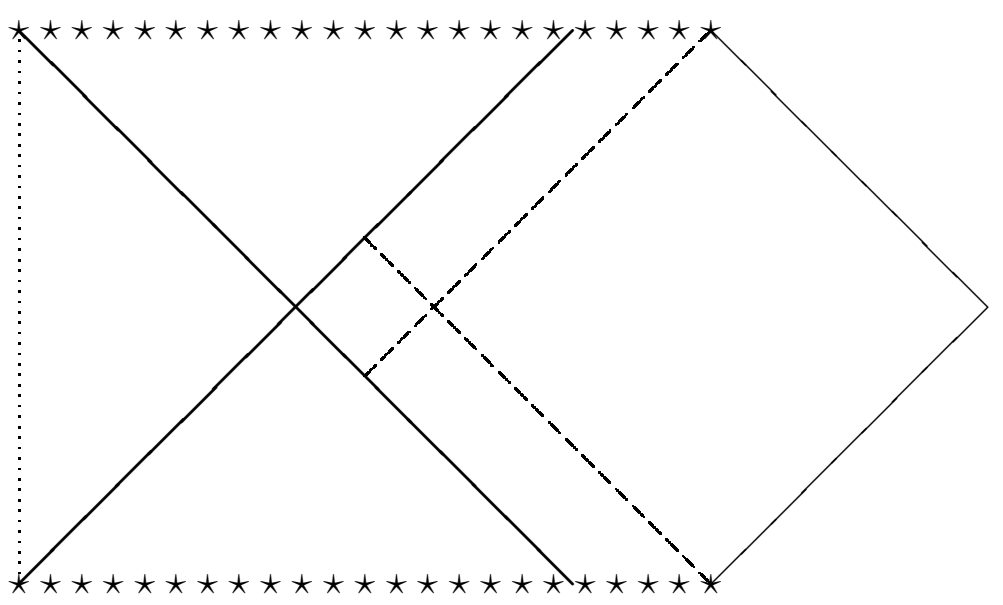

Figure 9: Penrose diagram of subcase $\mathrm{C}_{--}$. The dotted line is the regular center, the star lines are the singularities, the dashed lines are Schwarzschild horizons and the thick lines are the shells. 
Eq. (5) shows that some combinations of the two vertex coordinates is determined by the masses and cannot serve as independent parameters. In case $\mathrm{C}_{a b}$, if $a \neq 0$ and $b \neq 0$, we have

$$
\begin{aligned}
v_{l}-u_{l} & =2 r \\
b v_{d}-u_{d} & =4 M_{d} \ln \left[b \kappa^{-1}\left(\frac{r}{2 M_{d}}\right)\right], \\
v_{u}-a u_{u} & =4 M_{u} \ln \left[a \kappa^{-1}\left(\frac{r}{2 M_{u}}\right)\right], \\
b v_{r}-a u_{r} & =4 M_{r} \ln \left[a b \kappa^{-1}\left(\frac{r}{2 M_{r}}\right)\right],
\end{aligned}
$$

where $r$ is given by Eq. (8). Hence, only one parameter of each pair $\left(u_{K}, v_{K}\right)$ is free (if it is finite), and then it runs through the whole range $(-\infty, \infty)$ within each case.

Let us turn to the question which parameter is to be chosen for the purpose of providing information about the position of the shell pair with respect to infinity. The most straightforward choice is either $u_{r}$ or $v_{r}$. Clearly, $u_{r}$ and $v_{r}$ are even directly observable from infinity as retarded or advanced time coordinates of the out-going or in-going shell, as long as the shells are not bound or marginally bound.

It seems, however, that a conflict between two different notions of observability emerges here. On the one hand, there is observability in the canonical sense where the whole Cauchy surface is observable, and on the other hand there is observability from the asymptotic infinity. This does not concern the non-geometric observables only. Even some geometric observables seem not to be observable or manipulable from the infinity in the bound cases. For example, $b v_{d}-u_{d}(b=-)$ is not observable in cases $\mathrm{C}_{0-} \mathrm{C}_{+-} \mathrm{C}_{--}$. This is an interesting point that must have some relevance for our program of reducing the collapse to a scattering problem. It cannot, however, be dealt with in the present paper; only the observability in the canonical sense is considered here.

In the generic cases $a \neq 0$ and $b \neq 0$, both $u_{r}$ and $v_{d}$ are regular and any of them can be chosen. They are related by

$$
v_{r}=a b u_{r}+4 a M_{r} \ln \left[a b \kappa^{-1}\left(\frac{r}{2 M_{r}}\right)\right],
$$

where $r$ is defined by Eq. (8). In addition, $u_{r}$ is well-defined in the cases $\mathrm{C}_{+0}$ and $\mathrm{C}_{-0}, v_{r}$ in $\mathrm{C}_{0+}$ and $\mathrm{C}_{0-}$. If we exclude the case $\mathrm{C}_{00}$, then the rest of the physical phase space can be covered by two coordinate charts. The first chart is defined by the functions $M_{d}, M_{u}, M_{r}$ and $u_{r}$; it covers the cases $\mathrm{C}_{++}, \mathrm{C}_{+0}, \mathrm{C}_{+-}, \mathrm{C}_{-+}, \mathrm{C}_{-0}$ and $\mathrm{C}_{--}$. The second is defined by $M_{d}, M_{u}, M_{r}$ and $v_{r}$ and covers the cases $\mathrm{C}_{++}$, $\mathrm{C}_{0+}, \mathrm{C}_{+-}, \mathrm{C}_{-+}, \mathrm{C}_{0-}$ and $\mathrm{C}_{--}$. In the overlapping regions $\mathrm{C}_{++}, \mathrm{C}_{+-}, \mathrm{C}_{-+}$and $\mathrm{C}_{--}$, there is a unique transformation. In cases $\mathrm{C}_{0+}, \mathrm{C}_{+0}, \mathrm{C}_{0-}$ and $\mathrm{C}_{-0}$ we have only one choice because one of the two parameters diverges. 
In case $\mathrm{C}_{00}$, both parameters $u_{r}$ and $v_{r}$ diverge. From the point of view of infinity, all solutions of the case are static; their position with respect to a frame at infinity is not defined: no property measurable at infinity changes if the frame at infinity is time shifted. However, complete solutions of case $\mathrm{C}_{00}$ do not possess any additional symmetry in comparison to other cases because $\mathcal{M}_{l}, \mathcal{M}_{u}$ and $\mathcal{M}_{d}$ do not. The constraint surface of the ADM formalism is regular (cf. 16]), and there is a well defined pull back of the symplectic form to the constraint surface there.

A regular coordinate system in the phase space is constructed in paper III. It cannot be constructed in the present paper because it has to fulfill more conditions than just boundedness of the coordinate functions at all points of the space. The most important is of course the regularity of the symplectic form. We certainly could replace $v_{r}$ by functions of $v_{r}, M_{d}, M_{u}$ and $M_{r}$ that would be regular at $\mathrm{C}_{00}$, but there is no point in doing this until we know the symplectic form.

\section{Choice of gauge}

By a choice of gauge, a unique background manifold $\mathrm{M}$ is specified and each solution can be described as a set of fields and branes on $\mathrm{M}$, dependent on both the physical phase space coordinates and those on $\mathrm{M}$ (cf. [8] and [9]). The fields have to satisfy certain conditions. Thus, for the case of gravitating shells, the metric is required to be continuous, piecewise smooth and to satisfy some boundary conditions at the regular center and at the infinity $([9])$. A continuous metric determines uniquely a class of $C^{1}$ coordinate systems. Any gauge is required to be $C^{1}$. In this sense, no gauge has been chosen as yet.

A point important for the subsequent calculations is that there is a gauge choice for each case and that there is a well defined transformation between such gauge and the description of solutions of the case as given in the preceding section. This transformation is singular at some points (some transformation functions are divergent and some have step discontinuities), and it depends on more parameters that the dimension of the physical phase space has. However, the transformation is, locally, very much like a gauge transformation. Let us show it for case A.

Our construction of a regular gauge is based on the following interesting property of the DNEF coordinates.

Lemma 2 Let $p$ be a point of a shell, let $\left(X_{1}, Y_{1}\right)$ be some DNEF coordinate pair in a neighborhood $\mathcal{U}$ of $p$ left from the shell and $\left(X_{2}, Y_{2}\right)$ that right from the shell. Let the shell points in $\mathcal{U}$ satisfy the equations $X_{1}=X_{1}(p)$ and $X_{2}=X_{2}(p)$. Let the Schwarzschild radial coordinate $R=R_{n}\left(X_{n}, Y_{n}\right)$ satisfy $R_{n}\left(X_{n}, Y_{n}\right) \neq 2 M_{n}$ in $\mathcal{U}$ for 
both $n=1,2$. Let, finally, the functions $X$ and $Y$ be defined right from the shell by

$$
X=X_{2}-X_{2}(p)+X_{1}(p)
$$

and

$$
R_{2}\left(X_{2}(p), Y_{2}(Y)\right)=R_{1}\left(X_{1}(p), Y\right),
$$

and left from the shell by

$$
X=X_{1}, \quad Y=Y_{1}
$$

Then the functions $X$ and $Y$ form a $C^{1}$ coordinate system in $\mathcal{U}$.

Proof An observation that is crucial for the proof is the following: $X_{1}$ and $X_{2}$ must always be Eddington-Finkelstein functions of the same type. This follows immediately from the argument of matching divergences. Hence, there are four cases. These cases define the sign $\zeta$ by the following table:

\begin{tabular}{|c|c|c|}
\hline$X_{1}$ & $X_{2}$ & $\zeta$ \\
\hline$U_{1}^{+}$ & $U_{2}^{+}$ & + \\
$U_{1}^{-}$ & $U_{2}^{-}$ & - \\
$V_{1}^{+}$ & $V_{2}^{+}$ & - \\
$V_{1}^{-}$ & $V_{2}^{-}$ & + \\
\hline
\end{tabular}

Let us first consider the usual Eddington-Finkelstein coordinates $X_{n}$ and $R$ to both sides of the shell; the metric is

$$
d s^{2}=-\left(1-\frac{2 M_{n}}{R}\right) d X_{n}^{2}-2 \zeta d X_{n} d R+R^{2} d \Omega^{2} .
$$

The transformation to $\left(X_{n}, Y_{n}\right)$ can be performed by setting $R=R_{n}\left(X_{n}, Y_{n}\right)$ and we obtain in this way

$$
d s^{2}=-\left[\left(1-\frac{2 M_{n}}{R}\right)+2 \zeta \frac{\partial R_{n}}{\partial X_{n}}\right] d X_{n}^{2}-2 \zeta \frac{\partial R_{n}}{\partial Y_{n}} d X_{n} d Y_{n}+R_{n}^{2} d \Omega^{2} .
$$

It follows then that

$$
\frac{\partial R_{n}}{\partial X_{n}}=-\frac{\zeta}{2}\left(1-\frac{2 M_{n}}{R}\right)
$$

and

$$
\frac{\partial R_{n}}{\partial Y_{n}}=\frac{\zeta}{2} A_{n},
$$

where $A_{n}$ appears in the $\left(X_{n}, Y_{n}\right)$ metric:

$$
d s^{2}=-A_{n} d X_{n} d Y_{n}+R_{n}^{2} d \Omega^{2} .
$$


Eq. (19) implies

$$
\left(\frac{\partial R_{1}}{\partial Y_{1}}\right)_{\text {shell, }, Y_{1}=Y}=\left(\frac{\partial R_{2}}{\partial Y_{2}}\right)_{\text {shell }} \frac{\partial Y_{2}}{\partial Y}
$$

or

$$
\frac{\partial Y_{2}}{\partial Y}=\frac{\left(\frac{\partial R_{1}}{\partial Y_{1}}\right)_{\text {shell, } Y_{1}=Y}}{\left(\frac{\partial R_{2}}{\partial Y_{2}}\right)_{\text {shell }}} .
$$

Then, using Eq. (21), we have

$$
\frac{\partial Y_{2}}{\partial Y}=\frac{\left.A_{1}\right|_{\text {shell }}}{\left.A_{2}\right|_{\text {shell }}} .
$$

The transformation (20) gives, left from the shell,

$$
d s^{2}=-A_{1}(X, Y) d X d Y+R_{1}^{2}(X, Y) d \Omega^{2} .
$$

Similarly, the transformation (18) and (19) leads to, right from the shell,

$$
d s^{2}=-\left.A_{2}(X, Y) \frac{A_{1}}{A_{2}}\right|_{\text {shell }} d X d Y+R_{2}^{2}(X, Y) d \Omega^{2} .
$$

Thus, the metric is continuous at the shell, because $R$ is, QED.

Our gauge for case A can be described as follows:

Construction of the $V$ coordinate in $\mathcal{M}_{l}, \mathcal{M}_{m}, \mathcal{M}_{r}$ : Let

$$
V:=V_{l}^{+}+v_{r 2}-v_{m 2}+v_{m 1}-v_{l 1}
$$

in $\mathcal{M}_{l}$,

$$
V:=V_{m}^{+}+v_{r 2}-v_{m 2}
$$

in $\mathcal{M}_{m}$ and

$$
V:=V_{r}^{+}
$$

in $\mathcal{M}_{r}$. Then $V$ is a continuous function in $\mathcal{M}$. The inverse transformation is

$$
V_{l}^{+}:=V-v_{r 2}+v_{m 2}-v_{m 1}+v_{l 1}
$$

for $V \in\left(-\infty, v_{r 2}-v_{m 2}+v_{m 1}\right)$,

$$
V_{m}^{+}:=V-v_{r 2}+v_{m 2}
$$

for $V \in\left(v_{r 2}-v_{m 2}+v_{m 1}, v_{r 2}\right)$ and

$$
V_{r}^{+}:=V
$$

for $V \in\left(v_{r 2}, \infty\right)$.

The construction of the $U$ coordinate is more laborious: 
Construction of the $U$ coordinate in $\mathcal{M}_{l}$ : In the left region, we set

$$
U:=U_{l}^{+}+v_{r 2}-v_{m 2}+v_{m 1}-v_{l 1}
$$

and the metric is given by

$$
A_{l}(U, V)=1
$$

and

$$
R_{l}(U, V)=\frac{-U+V}{2}
$$

Construction of the $U$ coordinate in $\mathcal{M}_{m}$ : Across the first shell, we must have

$$
R_{l}\left(U, V_{l}^{+}=v_{l 1}\right)=R_{m}\left(U_{m}^{+}, V_{m}^{+}=v_{m 1}\right)
$$

for $U_{m}^{+} \in(-\infty, \infty)$. Using Eq. (5), we obtain the relation between $U$ and $U_{m}^{+}$along this part of the shell:

$$
U=v_{r 2}-v_{m 2}+v_{m 1}-4 M_{m} \kappa\left[\exp \left(\frac{-U_{m}^{+}+v_{m 1}}{4 M_{m}}\right)\right] .
$$

Along the part $U_{m}^{-} \in\left(-\infty,-v_{m 1}\right)$ of the first shell, we obtain in an analogous way:

$$
U=v_{r 2}-v_{m 2}+v_{m 1}-4 M_{m} \kappa\left[-\exp \left(\frac{U_{m}^{-}+v_{m 1}}{4 M_{m}}\right)\right] .
$$

Let us use the right hand sides of Eqs. (28) and (29) as the definitions of the coordinate $U$ everywhere in $\mathcal{M}_{m}$. Then $U$ is continuous across the first shell. The inverse transformation is

$$
U_{m}^{+}(U)=v_{m 1}-4 M_{m} \ln \left[\kappa^{-1}\left(\frac{-U+v_{r 2}-v_{m 2}+v_{m 1}}{4 M_{m}}\right)\right]
$$

for $U \in\left(-\infty, U\left(H_{m}\right)\right)$, where $H_{m}$ is the Schwarzschild horizon in $\mathcal{M}_{m}$, and

$$
U_{m}^{-}(U)=v_{m 1}-4 M_{m} \ln \left[-\kappa^{-1}\left(\frac{-U+v_{r 2}-v_{m 2}+v_{m 1}}{4 M_{m}}\right)\right]
$$

for $U \in\left(U\left(H_{m}\right), v_{r 2}-v_{m 2}+v_{m 1}\right) . U\left(H_{m}\right)$ can easily be calculated from Eq. (28) by setting $U_{m}^{+}=+\infty$ yielding the result $U\left(H_{m}\right)=v_{r 2}-v_{m 2}+v_{m 1}-4 M_{m}$. If we substitute either Eqs. (23) and (30) or Eqs. (23) and (31) into Eq. (5), we obtain in both cases that

$$
R_{m}=2 M_{m} \kappa\left[\exp \left(\frac{V-v_{r 2}+v_{m 2}-v_{m 1}}{4 M_{m}}\right) \kappa^{-1}\left(\frac{-U+v_{r 2}-v_{m 2}+v_{m 1}}{4 M_{m}}\right)\right] .
$$


The metric in $\mathcal{M}_{m}$ can be written in the form

$$
d s^{2}=-A_{m}(U, V) d U d V+R_{m}^{2}(U, V) d \Omega^{2} ;
$$

it is obtained from the Eddington-Finkelstein line element

$$
d s^{2}=-\left(1-\frac{2 M_{m}}{R}\right) d V^{2}+2 d V d R+R^{2} d \Omega^{2}
$$

by the transformation $R=R_{m}(U, V)$. This implies that

$$
A_{m}=-2 \frac{\partial R_{m}}{\partial U}
$$

and

$$
\frac{\partial R_{m}}{\partial V}=\frac{1}{2}\left(1-\frac{2 M_{m}}{R_{m}}\right) .
$$

Eqs. (32)-(34) determine the metric in $\mathcal{M}_{m}$ as function of the coordinates $U$ and $V$ and parameters $M_{m}, v_{r 2}$ and $v_{m 2}-v_{m 1}$.

Construction of the $U$ coordinate in $\mathcal{M}_{r}$ : The coordinate $U$ is determined in $\mathcal{M}_{r}$ from the condition of continuity across the second shell,

$$
R_{m}\left(U, v_{r 2}\right)=R_{r}\left(U_{r}^{+}, v_{r 2}\right)
$$

for $U_{r}^{+} \in(-\infty, \infty)$, and

$$
R_{m}\left(U, v_{r 2}\right)=R_{r}\left(U_{r}^{-}, v_{r 2}\right)
$$

for $U_{r}^{-} \in\left(-\infty,-v_{r 2}\right)$, where $R_{r}$ is the Schwarzschild radial coordinate in $\mathcal{M}_{r}$. A procedure analogous to that for $\mathcal{M}_{m}$ results in the transformation formulae:

$$
\begin{aligned}
& U_{r}^{+}(U)=v_{r 2}- \\
& \quad 4 M_{r} \ln \left(\kappa^{-1}\left\{\frac{M_{m}}{M_{r}} \kappa\left[\exp \left(\frac{v_{m 2}-v_{m 1}}{4 M_{m}}\right) \kappa^{-1}\left(\frac{-U+v_{r 2}-v_{m 2}+v_{m 1}}{4 M_{m}}\right)\right]\right\}\right)
\end{aligned}
$$

for $U \in\left(-\infty, U\left(H_{r}\right)\right)$, and

$$
\begin{aligned}
& U_{r}^{-}(U)=-v_{r 2}+ \\
& 4 M_{r} \ln \left(-\kappa^{-1}\left\{\frac{M_{m}}{M_{r}} \kappa\left[\exp \left(\frac{v_{m 2}-v_{m 1}}{4 M_{m}}\right) \kappa^{-1}\left(\frac{-U+v_{r 2}-v_{m 2}+v_{m 1}}{4 M_{m}}\right)\right]\right\}\right)
\end{aligned}
$$

for $U \in\left(U\left(H_{r}\right), U_{0 r}\right)$, where $H_{r}$ is the Schwarzschild horizon in $\mathcal{M}_{r}$, and $U_{0 r}$ is the value of the coordinate $U$ for which the second shell has zero radius,

$$
U\left(H_{r}\right)=v_{r 2}-v_{m 2}+v_{m 1}-4 M_{m} \kappa\left[\exp \left(\frac{-v_{m 2}+v_{m 1}}{4 M_{m}}\right) \kappa^{-1}\left(\frac{M_{r}}{M_{m}}\right)\right]
$$


and

$$
U_{0 r}=v_{r 2}-v_{m 2}+v_{m 1}-4 M_{m} \kappa\left[-\exp \left(\frac{-v_{m 2}+v_{m 1}}{4 M_{m}}\right)\right] .
$$

Eqs. (5), (24), (36) and (37) imply the following line element:

$$
d s^{2}=-A_{r}(U, V) d U d V+R_{r}^{2}(U, V) d \Omega^{2},
$$

where

$$
\begin{aligned}
& R_{r}(U, V)=2 M_{r} \kappa\left(\exp \left\{\frac{V-v_{r 2}}{4 M_{r}}\right\} \times\right. \\
& \left.\kappa^{-1}\left\{\frac{M_{m}}{M_{r}} \kappa\left[\exp \left(\frac{v_{m 2}-v_{m 1}}{4 M_{m}}\right) \kappa^{-1}\left(\frac{-U+v_{r 2}-v_{m 2}+v_{m 1}}{4 M_{m}}\right)\right]\right\}\right)
\end{aligned}
$$

for $U \in\left(-\infty, U_{0 r}\right)$

$$
A_{r}(U, V)=-2 \frac{\partial R_{r}}{\partial U}
$$

and the identity

$$
\frac{\partial R_{r}}{\partial V}=\frac{1}{2}\left(1-\frac{2 M_{r}}{R_{r}}\right)
$$

is also valid. One easily verifies that the function $A(U, V)$ defined by $A(U, V)=$ $A_{i}(U, V)$ in $\mathcal{M}_{i}$ is continuous across the shells. The function $R(U, V)$ defined by $R(U, V)=R_{i}(U, V)$ in $\mathcal{M}_{i}$ is continuous by construction. The background manifold $\mathrm{M}$ can be defined by the domains of coordinates $U$ and $V$ :

$$
U \in(-\infty, \infty), \quad V \in(U, \infty)
$$

the regular center part of the boundary is $V=U, U=-\infty$ is $\mathcal{I}^{-}$and $V=\infty$ is $\mathcal{I}^{-}$. The solutions are described as metric fields and shell trajectories on $\mathrm{M}$ that depend on the parameters $M_{m}, M_{r}, v_{r 2}$ and $v_{m 2}-v_{m 1}$.

The transformation between the coordinates $U$ and $V$ on one hand and $U_{l}^{+}, V_{l}^{+}$, $U_{m}^{ \pm}, V_{m}^{+}, U_{r}^{ \pm}$and $V_{r}^{+}$on the other is given by Eqs. (22)-(24), (25), (30), (31), (36) and (37). They depend on additional parameters $v_{m 1}$ and $v_{l 1}$.

We observe that the transformation functions have step discontinuity at $V=v_{r 2}-$ $v_{m 2}+v_{m 1}$ and $V=v_{r 2}$, they are divergent at $U=U\left(H_{m}\right)$ for $V \in\left(v_{r 2}-v_{m 2}+v_{m 1}, v_{r 2}\right)$ and at $U=U\left(H_{r}\right)$ for $V \in\left(v_{r 2}, \infty\right)$. In all other points, they represent a regular, parameter-dependent gauge transformation. We shall, therefore, call coordinates similar to $U_{l}^{+}, V_{l}^{+}, U_{m}^{ \pm}, V_{m}^{+}, U_{r}^{ \pm}$and $V_{r}^{+}$singular gauge. One might be able to use singular gauges for calculation of some gauge-invariant quantities. In fact, we shall do so in the subsequent papers, II and III. 


\section{Symmetries}

In Sec. 2, a complete account of the space of solutions has been provided. In the present section, we are going to describe some relations between different solutions in this space. First, we observe that some solutions are isometric to each other. The isometries are of two types; time shift and time reversal. Second, there are also conformally related solutions. The time shifts and dilatations generate one-dimensional subfamilies of solutions; the time reversal acts only inside pairs of solutions. The symmetries are interesting for us mainly because they will be used for a construction of the most interesting observables (such as, e.g., the Hamiltonian) in the quantum theory (cf. [10])

The fact that physically different solutions are isometric may need some comment: how can two isometric solutions be physically different? The cause of such a difference is the asymptotically flat region. We consider just one family of asymptotic observers common for the whole space of solutions. For these observers, two shells such that one is sent earlier than the other represent two physically different situations even if the shells are completely isomorphic in all other properties. In fact, the time shifts and the time reversal is the only remnant of the Bondi-MetznerSachs (BMS) group $G_{\mathrm{BMS}}$ that is non-trivial and preserves the spherical symmetry of our model. In a general situation with asymptotically flat region, the whole BMS group of transformations between standard asymptotic frames acts as a group of symmetry. Indeed, the full diffeomorphism group $G$ (each element of which sends any solution isometrically to another one, see [9]) can be split into the gauge and the symmetry groups. A subgroup $G_{0}$ of $G$ that leaves, roughly speaking, all points at the infinity invariant ( $G_{0}$ has to be selected by a suitable fall-off condition) is the gauge group; the factor group $G / G_{0}=G_{\mathrm{BMS}}$ is the group of symmetry.

Each symmetry $\Phi$ has well-defined actions in different spaces of interest. First, $\Phi$ acts in the space of parameters that describe the solutions. The action just tells us which pairs of solutions are related by $\Phi$. Next, if a gauge is chosen, $\Phi$ also acts on the corresponding background manifold. This action tells us which points of the two $\Phi$-related solution spacetimes are mapped on each other by the corresponding diffeomorphism. Third, $\Phi$ acts in the asymptotic space that is common to all solutions and that represents the view of the asymptotic observers. Fourth, $\Phi$ also acts on the phase space of the system. In fact, the first action is nothing but the action on the physical (reduced) phase space; one only has to equip the space of parameters with a symplectic structure. But there will also be an action on the constraint surface, the points of which are initial data for solutions, or even on the extended phase space. In this section, we shall study only the first three actions. 


\subsection{Time shift}

Time shifts form a one-dimensional group of transformations. Let the group parameter be denoted by $t$. Time shifts preserve the in- and out-going character of the shells, so we can work case by case.

Consider case A. The coordinates on the physical phase space are $M_{m}, v_{m 2}-v_{m 1}$, $M_{3}$ and $v_{r 2}$, The three variables $M_{m}, v_{m 2}-v_{m 1}$ and $M_{r}$ determine the geometry uniquely. Thus, solutions that do not differ in the values of these parameters are isometric. Let us define the action $\Phi_{t}^{P}$ of the $t$-shift isometry $\Phi_{t}$ on the physical phase space by

$$
\Phi_{t}^{P}\left(M_{m}, v_{m 2}-v_{m 1}, M_{r}, v_{r 2}\right)=\left(M_{m}, v_{m 2}-v_{m 1}, M_{r}, v_{r 2}+t\right) .
$$

This definition is suggested by the nature of the parameter $v_{r 2}$. As the advanced time of the external shell, it is measurable by the asymptotic observers and it is shifted by $t$ if the asymptotic observers shift their clocks by $-t$.

In Sec. 3, a particular gauge is chosen for case A. A background manifold $\mathrm{M}$ covered by coordinates $U$ and $V$ is well defined, and each solution appears as a particular metric field

$$
d s^{2}=-A\left(U, V ; M_{m}, v_{m 2}-v_{m 1}, M_{r}\right) d U d V+R^{2}\left(U, V ; M_{m}, v_{m 2}-v_{m 1}, M_{r}\right) d \Omega^{2}
$$

on $\mathrm{M}$, where the functions $A$ and $R$ are defined in Sec. 3 by Eqs. (26), (27), (32), (34), (39) and (40). The trajectories of the shells in $M$,

$$
V=v_{r 2}-v_{m 2}-v_{m 1}
$$

and

$$
V=v_{r 2}
$$

also depend on the parameters. Hence, there must be a unique map $\Phi_{t}^{\mathrm{M}}: \mathrm{M} \mapsto \mathrm{M}$ for each $t$ such that the metric and the shell trajectories given by the values of the parameters $M_{m}, v_{m 2}-v_{m 1}, M_{r}$ and $v_{r 2}$ are mapped into the metric and trajectories for $M_{m}, v_{m 2}-v_{m 1}, M_{r}$ and $v_{r 2}+t$. This is done by the map

$$
\Phi_{t}^{\mathrm{M}}(U, V)=(U+t, V+t) .
$$

Indeed, Eqs. (26), (27), (32), (34), (39) and (40) show that the functions $A$ and $R$ depend on $U, V$ and $v_{r 2}$ only through the combinations $-U+V,-U+v_{r 2}$ and $V-v_{r 2}$. The shells given by Eqs. (42) and (43) are also shifted properly by (44). Similar map $\Phi_{t}^{\mathrm{M}}$ can be found for all cases if a gauge is chosen. 
The structure that we need near $i^{0}$ is just the time measured by the asymptotic observers; the range of this time is $(-\infty, \infty)$. We have to define such a coordinate in each solution. The Schwarzschild time coordinate $T_{\infty}$ in $\mathcal{M}_{r}$ for all cases A, A', B, B', C, C' seems to be a good candidate. It must be defined in $\mathcal{M}_{r}$ by fixing it there with respect to the already chosen coordinates $\left(U_{r}^{+}, V_{r}^{+}\right)$. This can be done as follows:

$$
T_{\infty}:=\frac{U_{r}^{+}+V_{r}^{+}}{2} ;
$$

Above definition of the asymptotic coordinate $T_{\infty}$ in each solution is formally analogous to choice of gauge; it is also similarly non unique. However, all mathematically different choices of asymptotic time give equivalent descriptions of the physical properties of the system.

Consider Case A and two solutions, one given by the values $M_{m}, v_{m 2}-v_{m 1}, M_{r}$ and $v_{r 2}$ of the parameters and the other by $M_{m}, v_{m 2}-v_{m 1}, M_{r}$ and $v_{r 2}+t$. Their $\mathcal{M}_{r}$-parts are mapped isometrically on each other by $\Phi_{t}$ as follows

$$
U_{r}^{+} \mapsto U_{r}^{+}+t, \quad V_{r}^{+} \mapsto V_{r}^{+}+t
$$

Hence,

$$
T_{\infty} \mapsto T_{\infty}+t
$$

This defines the action $\Phi_{t}^{\infty}$ of $\Phi_{t}$ at the infinity.

\subsection{Time reversal}

Consider the solution of case A determined by the values $M_{m}, v_{m 2}-v_{m 1}, M_{r}$ and $v_{r 2}$ of the parameters and the solution of case B determined by $M_{m}^{\prime}, u_{m 2}-u_{m 1}$, $M_{r}^{\prime}$ and $u_{r 2}$. Suppose that $M_{m}^{\prime}=M_{m}, M_{r}^{\prime}=M_{r}, u_{m 2}-u_{m 1}=-\left(v_{m 2}-v_{m 1}\right)$ and $u_{r 2}=-v_{r 2}$. Then the two solutions are isometric and the corresponding map inverts time orientation. This motivates the definition

$$
\mathbf{T}^{P}\left(M_{m}, v_{m 2}-v_{m 1}, M_{r}, v_{r 2}\right)=\left(M_{m},-\left(v_{m 2}-v_{m 1}\right), M_{r},-v_{r 2}\right)
$$

of the action $\mathbf{T}^{P}$ of the time reversal $\mathbf{T}$ on the physical phase space of cases $\mathrm{A}$ and B. The inverse map is easily to calculate.

Similarly, for the case $\mathrm{C}$, we define for the charts $\left(M_{d}, M_{u}, M_{r}, v_{u}\right)$ in the physical phase space of case $\mathrm{C}$ and $\left(M_{d}^{\prime}, M_{u}^{\prime}, M_{r}^{\prime}, u_{d}^{\prime}\right)$ in that of case $\mathrm{C}^{\prime}$ :

$$
\mathbf{T}^{P}\left(M_{d}, M_{u}, M_{r}, v_{u}\right)=\left(M_{u}, M_{d}, M_{r},-v_{u}\right) .
$$

Consider the action of the time reversal on the background manifold M. One can choose the gauge in all cases so that always

$$
\mathrm{T}^{\mathrm{M}}(U, V)=(-V,-U)
$$


Finally, with our definition (45) of the asymptotic time $T_{\infty}$, we clearly have

$$
\mathbf{T}^{\infty}\left(T_{\infty}\right)=-T_{\infty}
$$

\subsection{Dilatation}

Consider again the formulae (26), (27), (32), (34), (39) and (40) of case A. The transformation

$$
\begin{array}{rlrl}
M_{m} & \mapsto e^{\lambda} M_{m}, & M_{r} \mapsto e^{\lambda} M_{r}, \\
v_{m 2}-v_{m 1} \mapsto e^{\lambda}\left(v_{m 2}-v_{m 1}\right), & v_{r 2} \mapsto e^{\lambda} v_{r 2},
\end{array}
$$

together with

$$
U \mapsto e^{\lambda} U, \quad V \mapsto e^{\lambda} V
$$

lead to $R \mapsto e^{\lambda} R$ and $A \mapsto A$, so that the metric is rescaled as follows

$$
-A d U d V+R^{2} d \Omega^{2} \mapsto e^{2 \lambda}\left(-A d U d V+R^{2} d \Omega^{2}\right)
$$

The asymptotic time $T_{\infty}$ (see Eq. (45)) is then transformed so that $T_{\infty} \mapsto e^{\lambda} T_{\infty}$.

Analogous equations hold for all other cases. Eqs. (47) and (48) describe the action of the dilatation group $D_{\lambda}$ on the space of the parameters and Eq. (49) that on the background manifold.

The symmetry of the model under dilatation is, of course, due to the shell matter being light-like.

\section{Acknowledgments}

The authors are thankful for useful discussions by T. Dray, W. Israel, K. V. Kuchař and P. Minkowski. The work has been supported by the Swiss Nationalfonds and by the Tomalla Foundation, Zurich.

\section{References}

[1] P. Bergmann, Rev. Mod. Phys. 33 (1961) 510.

[2] C. Torre, Phys. Rev. D46 (1993) R3231.

[3] P. Hájíček and C. Isham, J. Math. Phys. 37 (1996) 3522.

[4] P. Hájíček, J. Math. Phys. 36 (1995) 4612, Class. Quant. Grav. 13 (1996) 1353. 
[5] B. S. DeWitt, Dynamical Theory of Groups and Fields, in Relativity, Groups and Topology I, eds. C. DeWitt and B. S. DeWitt, Gordon and Breach, London (1964).

[6] A. Ashtekar, Asymptotic Quantization, Bibliopolis, Napoli (1987)

[7] K. V. Kuchar, J. Math. Phys. 13 (1972) 768.

[8] P. Hájíček and J. Kijowski, Phys. Rev. D 61 (2000) 024037.

[9] P. Hájíček and C. Kiefer, Nucl. Phys. B 603 (2001) 491.

[10] P. Hájíček, Nucl. Phys. B 603 (2001) 555.

[11] J. Bicak and P. Hájíček, Phys. Rev. 56 (1997) 4706.

[12] C. Barrabes and W. Israel, Phys. Rev. D 43 (1991) 1129.

[13] T. Dray and G. t’Hooft, Commun. Math. Phys. 99 (1985) 613.

[14] I. H. Redmount, Progr. Theor. Phys. 73 (1995) 1401.

[15] E. Witten, Comm. Math. Phys. 80 (1981) 381.

[16] J. Marsden, Lectures on Geometric Methods in Mathematical Physics, CMBS Vol. 37, SIAM Philadelphia, 1981. 